\title{
Weak Signal Detection Based on Stochastic Resonance of Piecewise Model
}

\author{
Wenli Zhao ${ }^{1, a}$, Yuanping Yin ${ }^{1, b}$, Qian Fang ${ }^{1, c}$ \\ ${ }^{1}$ College of Mechanical Engineering, Hangzhou Dianzi University, Hangzhou 310018,China \\ azhaowlcn@yahoo.com.cn, byinyuanping23@163.com, fangqian326@163.com
}

Keywords: Stochastic Resonance, Piecewise model, Weak signal detection

\begin{abstract}
Based on traditional theory of bistable stochastic resonance, this paper puts forward a piecewise stochastic resonance model which used to detect medium- low-frequency weak periodic signal and deducts analytical results of signal-to-noise ratio. Besides, we have done simulation experiments and contrast analysis of the two models also. The result shows that the piecewise model is better to detect medium- low-frequency weak periodic signal in background of strong noise with higher SNR and wider frequency range. It has raised adaptability to detect periodic signal which is common in mechanical failure. Combined with the piecewise bistable stochastic resonance model and the modulate method, it is more effectively to achieve judgment and detection of the mechanical fault signals.
\end{abstract}

\section{Introduction}

Human beings have already developed a series of test methods and techniques in weak signal detection, such as correlation filtering technology, etc, but they all need to restrain noise. When noise was reduced, however, the signal was also damaged at the same time. The stochastic resonance has provided a useful method to extract weak signal in background of strong noise. But a lot of research represented by adiabatic approximation theory has shown that merely in small parameters condition (the signal amplitude, frequency and noise intensity are much less than 1), the system can produce stochastic resonance to detect the weak signal effectively[1-3]. However, in engineering practice such as mechanical failure, measured signal is medium-low-frequency periodic signal which is often overwhelmed by noise and this signal is much weaker than noise. So, the practical application of stochastic resonance method in engineering is limited.

The paper puts forward a piecewise stochastic resonance model which used to detect mediumlow-frequency weak periodic signal.

\section{The theory of stochastic resonance and the piecewise stochastic resonance}

The theory based on stochastic resonance in bistable system. The nonlinear bistable system is one of typical stochastic resonance models. Its essence is an overdamped Brownian particle moving in a bistable potential well, accompanied by periodic driving force and noise. The system can be modeled with the Langevin equation of motion in the form:

$$
\dot{x}(t)=a x(t)-b x^{3}(t)+A \cos \Omega t+n(t)
$$

Where $a$ and $b$ are parameter of the system, $a>0, b>0 . x(t)$ is output of the system and $A \cos \Omega t$ is input signal, $\mathrm{A}$ and $\Omega$ are the amplitude and the frequency of the input signal respectively. In addition, there is an additive noise $n(t)$ with intensity $\mathrm{D}$. Where $\mathrm{A}=0$ and $\mathrm{D}=0$, the nonlinear bistable system is:

$$
\dot{x}(t)=a x(t)-b x^{3}(t)
$$

A simple symmetric bistable potential has a form of standard quartic:

$$
U(x)=-\frac{a}{2} x^{2}+\frac{b}{4} x^{4}
$$




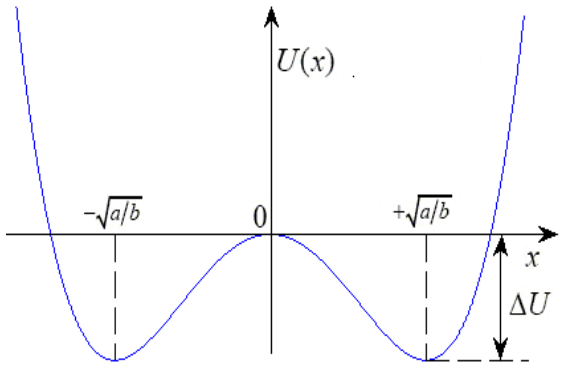

Fig. 1 The quartic bistable potential

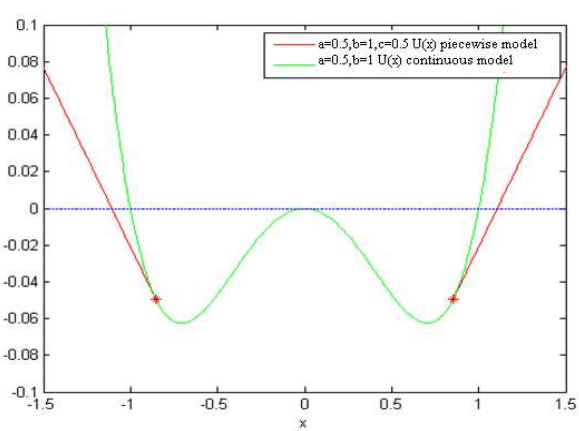

Fig. 2 The potential of the two models

The quartic bistable potential is shown in Fig. 1 [1].According to adiabatic approximation theory, under condition of small signal parameters, the system is possible to form synergy and produce stochastic resonance. But medium-low-frequency periodic signal and multi-frequency harmonic signal are common in mechanical failure, they are difficult to detect because they don't satisfy the condition of small parameters. In order to achieve effective detection, the piecewise bistable stochastic resonance model was established in Fig. 2. Through simulation, it is confirmed that the system can broaden frequency band effectively in order to increase the frequency scanning efficiency. Combined with the modulate characteristic[5], the system can transform various frequency components into small parameter signals which can transfer noise energy to the useful signal energy successfully and achieve the judgment on detection of the mechanical fault signal effectively.

Model based on the piecewise bistable stochastic resonance. According the bistable stochastic resonance model, this paper puts forward a piecewise stochastic resonance model and its mathematical equations are:

$$
\dot{x}=\left\{\begin{array}{cc}
K+A \cos \Omega t+n(t) & x<-x_{0} \\
a x-b x^{3}+A \cos \Omega t+n(t) & -x_{0} \leq x \leq x_{0} \\
-K+A \cos \Omega t+n(t) & x>x_{0}
\end{array}\right.
$$

Where $a$ and $b$ are the parameter of the system, $a>0, b>0 . x(t)$ is the output of the system and $A \cos \Omega t$ is the input signal, $\mathrm{A}$ and $\Omega$ are the amplitude and the frequency of the input signal respectively. In addition, there is an additive noise $n(t)$ with intensity $\mathrm{D}=0$. Typically, this is white Gaussian noise respectively. $\left(\mathrm{x}_{0}, \mathrm{y}_{0}\right)$ is the coordinate of the tangency point, $\mathrm{K}$ is the slope of the tangency point and $\mathrm{C}$ is the adjust parameter of the tangency point.

$$
x_{0}=\mathrm{C} \sqrt{a / b}, y_{0}=-\frac{a}{2} x_{0}{ }^{2}+\frac{b}{4} x_{0}{ }^{4}=\frac{a^{2} C^{2}}{2 b}\left(\frac{C^{2}}{2}-1\right), \mathrm{K}=a x_{0}+b x_{0}{ }^{3}=\left(\mathrm{C}^{2}-1\right) a \mathrm{C} \sqrt{a / b}
$$

So, the potential of the piecewise stochastic resonance has the form:

$$
U(x)=\left\{\begin{array}{cc}
-K\left(x+x_{0}\right)+y_{0} & x<-x_{0} \\
-\frac{a x^{2}}{2}+\frac{b x^{4}}{4} & -x_{0} \leq x \leq x_{0} \\
K\left(x-x_{0}\right)+y_{0} & x>x_{0}
\end{array}\right.
$$

There is a unstable state at $x=0$ and two stable states at $x_{m}= \pm \sqrt{a / b}$, separated by a barrier of height $\Delta U=a^{2} / 4 b$ under static conditions.

Fig. 2 has shown potential of the two models. The red line has shown the piecewise stochastic resonance with parameters $a=0.5, b=1$ and $c=0.5$. The green line has shown the bistable potential with parameters $a=0.5$ and $b=1$.

For the bistable system, with the change of the potential function $U(x)$, coordinate value of the $x$ will become smaller, the output will be very easy to produce saturation phenomenon as shown in Fig. 2.Seen from Function 6 and Fig. 2, the piecewise stochastic resonance is better than the bistable 
stochastic resonance in terms of control. Because the piecewise model has a control parameter $\mathrm{C}$ compared with the bistable model. So, we can use parameters a and b to control the potential barrier height and parameter $\mathrm{C}$ to control the size of the openings which related to amplitude of output signal. Because the potential boundary of the piecewise model is linear, the system won't appear saturation phenomenon. Obviously, the piecewise model has an advantage than the bistable model. In order to facilitate analysis, we fixed parameter $b=1$ and $a=\mu$.

\section{Signal-to-noise Ratio}

Equation of one-dimensional bistable system and its solution. For the one-dimensional bistable system as Fig. 1, we can establish its probability equation as:

$$
\left\{\begin{array}{l}
\frac{d P_{-}(t)}{d t}=-R_{-} P_{-}(t)+R_{+} P_{+}(t) \\
\frac{d P_{+}(t)}{d t}=-R_{+} P_{+}(t)+R_{-} P_{-}(t)
\end{array}\right.
$$

So the solution is:

$$
\left\{\begin{array}{l}
P_{-}(t)=\frac{1}{2}\left(1+\mathrm{e}^{-\left(R_{-}+R_{+}\right) t}\right) \\
P_{+}(t)=\frac{1}{2}\left(1-\mathrm{e}^{-\left(R_{-}+R_{+}\right) t}\right)
\end{array}\right.
$$

When reaching the steady-state, $R_{-}=R_{+}=R$, the above equation can be written as:

$$
\left\{\begin{array}{l}
P_{-}(t)=\frac{1}{2}\left(1+e^{-2 R t}\right) \\
P_{+}(t)=\frac{1}{2}\left(1-e^{-2 R t}\right)
\end{array}\right.
$$

So the probability current can be written as:

$$
\left\{\begin{array}{l}
p_{-}(t)=\dot{P}_{-}(t)=-R e^{-2 R t} \\
p_{+}(t)=\dot{P}_{+}(t)=+R e^{-2 R t}
\end{array}\right.
$$

We can obtain its probability current on the Frequency domain by taking Fourier transform as:

$$
\left\{\begin{array}{c}
|p(\omega)|=\left|p_{-}(\omega)\right|+\left|p_{+}(\omega)\right|=\frac{2 R}{\sqrt{(2 R)^{2}+\omega^{2}}} \\
\varphi(\omega)=\arctan \left(\frac{\omega}{2 R}\right)
\end{array}\right.
$$

The Kramer escapes rate formula [1]:

$$
R^{-1}=\frac{1}{D} \int_{-\infty}^{A} e^{-U(x) / D} d x \int_{-\infty}^{A} e^{U(x) / D} d x
$$

Where $\mathrm{R}$ knows as Kranmers escape rate is the rate of probability flowing into the unstable region. Put Eq. 6 to the Eq. 12, we can get:

$$
R_{+}^{-1}(t)=-\frac{2 \sqrt{2} \pi y_{0}}{k D \mu} e^{\frac{\mu^{2}}{4 D}}\left[1-\frac{\sqrt{2} k D \pi}{2 \mu y_{0}} e^{\frac{\mu^{2}}{4 D}}\right]
$$

Because of $\mathrm{D} \ll 1$, due to the steady state, we can get:

$$
\left|R_{-}\right|=\left|R_{+}\right|=\frac{\sqrt{2} k D \mu}{4 \pi y_{0}} e^{-\frac{\mu^{2}}{4 D}}
$$


The response of periodic driving and signal-to-noise ratio (SNR) [1].Assuming that the noise background contains periodic signal $A(t)=A_{0} \cos \Omega t$, when $A_{0}$ is very small, the bistable system response can be expressed as:

$$
\langle x(t)\rangle=\bar{x}(D) \cos (\Omega t-\bar{\varphi})
$$

When $\omega=\Omega$, it can be written as:

$$
\left\{\begin{array}{c}
\bar{x}(D)=\frac{A_{0}\left\langle x^{2}\right\rangle_{0}}{D} \frac{2 R}{\sqrt{(2 R)^{2}+\Omega^{2}}} \\
\bar{\varphi}(D)=\arctan \left(\frac{\Omega}{2 R}\right)
\end{array}\right.
$$

It is easy to known that the amplitude response is dependent on the noise changes. The power spectrum can obtain through the Fourier transform of autocorrelation function:

$$
S(\omega)=\int_{-\infty}^{+\infty}\langle x(t) x(t+\tau)\rangle \mathrm{e}^{-\mathrm{j} \omega \tau} d \tau
$$

When the periodic signal isn't input to the system, the output power spectrum can be written as:

$$
S_{N}^{0}(\omega)=\lim _{T \rightarrow \infty} \frac{1}{T}|p(\omega)|^{2}=4 R\left\langle x^{2}\right\rangle_{0} /\left(4 R^{2}+\omega^{2}\right)
$$

After input the periodic signal to the system, the output power spectrum can be written as:

$$
S(\omega)=(\pi / 2) \bar{x}^{2}(D)[\delta(\omega-\Omega)+\delta(\omega+\Omega)]+S_{N}(\omega)
$$

Here $S_{N}(\omega)=S_{N}^{0}(\omega)+0\left(A_{0}^{2}\right)$, so the signal-to-noise ratio is:

$$
S N R=2\left[\int_{0}^{\infty} S(\omega) d \omega\right] / S_{N}(\Omega)=\pi\left(A_{0} x_{m} / D\right)^{2} R
$$

For the continuous bistable system, it can obtain $x_{m}=\sqrt{\mu}$ and $R=\frac{\mu}{\sqrt{2} \pi} e^{-\frac{\mu^{2}}{4 D}}$. Put them into the above Equations, we can get:

$$
S N R=\frac{\sqrt{2} \mu^{2} A_{0}^{2} e^{-\frac{\mu^{2}}{4 D}}}{2 D^{2}}
$$

Fig. 3 has shown the relationship between the SNR and the noise intensity D in the continuous bistable system.

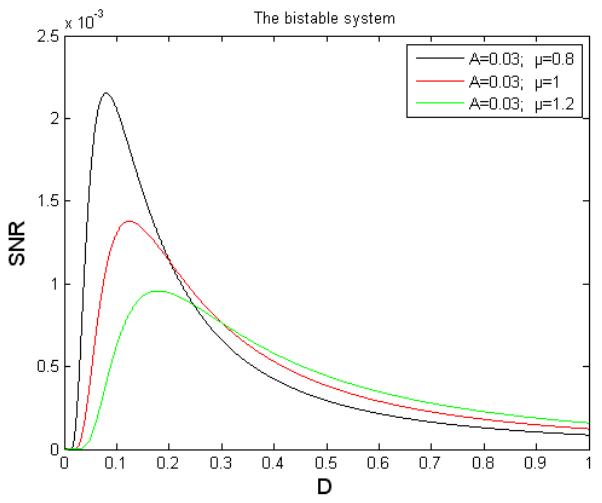

Fig. 3 The output SNR of the bistable system

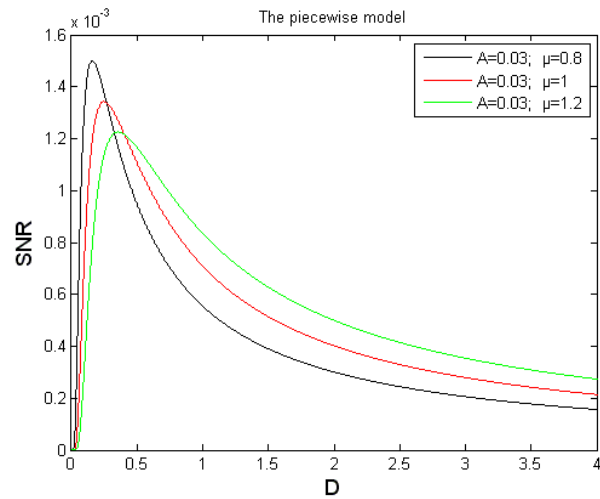

Fig. 4 The output SNR of the piecewise system

For the piecewise system, we can get $x_{m}=\sqrt{\mu}, R=\frac{\sqrt{2} k D \mu}{4 \pi y_{0}} e^{-\frac{\mu^{2}}{4 D}}$. Put it to Eq. 18, we can get:

$$
S N R=\pi\left(\frac{A_{0} x_{m}}{D}\right)^{2} R=\frac{\sqrt{2} A_{0}^{2} k \mu^{2}}{4 D y_{0}} \mathrm{e}^{-\frac{\mu^{2}}{4 D}}
$$

Fig. 4 has shown the relationship between the SNR and the noise intensity D in the piecewise system. 
Compared with output SNR of the two models, the piecewise model can meet the needs of the wider frequency range and strong noise effectively because it doesn't appear saturation.

\section{The simulation analysis}

Supposing the mixed signals $r(t)=A \cos \Omega t+n(t)$, where $A=0.6$ is the amplitude of the measurable signal and the frequency of the measurable signal is $f=0.01 \mathrm{~Hz}$ and $f=0.1 \mathrm{~Hz}$ respectively. In addition, there is a white Gaussian noise $n(t)$ with intensity D. First adjustment parameters $\mu=1$, the spectrum output waveform of the two models as follows:
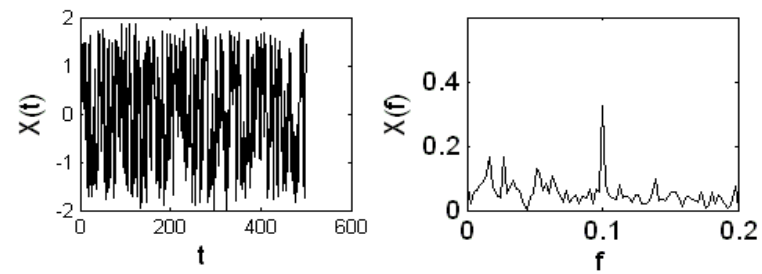

Fig. 5 The output of the bistable model: $\mathrm{f}=0.1 \mathrm{~Hz}$
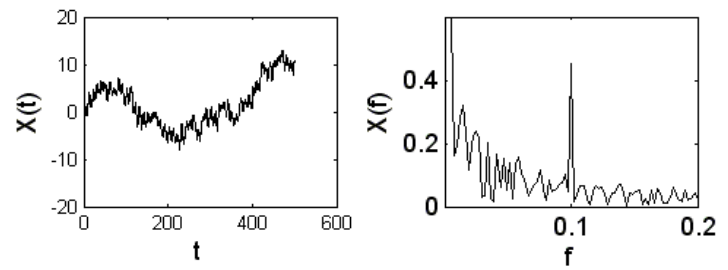

Fig. 6 The output of the piecewise model: $\mathrm{f}=0.1 \mathrm{~Hz}$

As an example, when the same noise and signal with the frequency $\mathrm{f}=0.1 \mathrm{~Hz}$ are put into the two systems respectively, the output waveform of the piecewise model has a better characteristics than the continuous bistable system. The other simulation test of the frequency has same effect. Thus, the piecewise model for low frequency signal detection has a better adaptability.

\section{Summary}

Through above analysis, extracting weak periodic signal in the background of strong noise, the piecewise model is controlled easily. The output SNR will be improved significantly and the range of frequency band is superior to the continuous bistable model. It has also improved adaptability of the periodic detection which is common in mechanical fault. Combined with the modulate method ${ }^{[5]}$, it is more efficiency in mechanical fault judgment and detection.

\section{Acknowledgment}

This paper is financially supported by the National Natural Science Foundation of China project (NO.50875070) and the Zhejiang Key Discipline of Marine Technology and Systems.

\section{References}

[1] Luca Gammaitoni, Peter Hanggi,Peter Jung, Fabio Marchesoni, Stochastic resonance, Reviews of Modern Physics(1998),Vol.70 (1).

[2] F. R. Palomo,J. M. Quero, L. G. Franquelo. Intermodulation distortion measures in a stochastic resonance[J]. IEEE International Symposium(2002), Vol.4(1),p.505-508.

[3]B.McNamara, K.Wiesenfeld. Theory of stochastic resonance. Phys.Rev.A(1989), Vol.39, p.4854-4869.

[4] M.I.Dykman, R.Mannella, PVE.McClintock, etal.Comment on "Stochastic Resonance in bistable systems". PrecedingComment, Phys.Rev.Lett(1990), Vol.65,p.2606.

[5] Wenli Zhao, Jin Liu, Yuanping Yin, Medium-low Frequency Signal Detection and Circuit Design Based on Stochastic Resonance Principle, Chinese Journal of Scientific Instrument(2011),Vol.32,( 4),p.721-728. 